\title{
Protocolo de Atención Ortodóncica en Pacientes con Trastorno del Espectro Autista (TEA)
}

\author{
Orthodontic Care Protocol for Patients with Autistic Spectrum Disorder (ASD)
}

Noemí Leiva Villagraํ \& Daniella Vergara Silva²

LEIVA,V. L. \& VERGARA, S. D. Protocolo de atención ortodóncica en pacientes con trastorno del espectro autista (TEA). Int. J. Odontostomat., 11(4):399-404, 2017.

RESUMEN: Actualmente se observa un aumento en el número de personas con necesidades especiales en atención en salud, dentro de ellos, aquellos pacientes diagnosticados con trastorno del espectro autista, quienes presentan altos requerimientos de tratamiento ortodóncico a causa de maloclusiones. A pesar de que padres y/o cuidadores se encuentran motivados a mejorar su calidad de vida mediante el mejoramiento de la estética dento-facial y la función oral, también son pacientes que no son tratados en clínicas convencionales dado alguna dificultad en el comportamiento que presenten en una atención odontólogica. Por la enorme dificultad que se puede encontrar en la atención clínica, el presente trabajo indica un protocolo de atención ortodóncica en pacientes con trastorno del espectro autista, útil para realizar una completa ayuda de cada paciente que permita un adecuado diagnóstico, plan de tratamiento y pronóstico en la especialidad de ortodoncia.

PALABRAS CLAVE: necesidades especiales, trastorno espectro autista, protocolo, ortodoncia.

\section{INTRODUCCIÓN}

La asociación americana de odontopediatría (AAPD, 2012) define necesidades especiales de atención en salud (NEAS) como toda condición o limitación física, del desarrollo, mental, sensorial, conductual, cognitiva o deterioro emocional o condición limitante que requiere tratamiento médico, intervención de atención de la salud, y/o el uso de servicios o programas especializados. La condición puede ser congénita, del desarrollo o adquirida a través de enfermedad, trauma, o causa medio ambiental y puede imponer limitaciones en la realización de actividades de auto-mantenimiento diario o limitaciones sustanciales en una actividad importante de la vida.

La atención odontológica de esta población en muchos casos requiere de equipamiento adecuado, profesionales con conocimientos especializados y a la vez técnicamente preparados para cumplir con una serie de cuidados específicos según el tipo de patología del paciente y sus necesidades únicas en salud para una atención de calidad (Dougall \& Fiske, 2008a).
Trastorno del Espectro Autista: El trastorno del espectro autista (TEA) es una condición compleja, diversa y multifactorial del desarrollo neurológico (Masi et al., 2017).

El diagnóstico de TEA se establece después de un cuidadoso examen médico, psicológico, neurológico, psiquiátrico y se basa en 4 criterios: alteraciones graves en las relaciones sociales; alteraciones graves en el desarrollo de la comunicación; patrones de comportamiento, intereses y actividades que son restringidos, repetitivos y estereotipados; y el inicio temprano (antes de 3-5 años de edad). No todos estos síntomas deben necesariamente estar presentes simultáneamente o con la misma intensidad. Uno de los principales problemas para lograr un diagnóstico definitivo se basa en la falta de pruebas genéticas, médicas o analíticas específicas (Abrahams \& Geschwind, 2008; APA, 2013).

Por lo tanto el diagnóstico está basado en la

\footnotetext{
${ }^{1}$ Especialista en Ortodoncia, Directora Unidad de Malformación Craneofacial, Facultad de Odontología, Universidad de Chile, Santiago, Chile. ${ }^{2}$ Cirujano Dentista Facultad de Odontología Universidad de Chile, Santiago, Chile.
} 
observación del comportamiento del niño sobre criterios centrados en impedimentos en la comunicación e interacción social, patrones restringidos y repetitivos de comportamiento, intereses o actividades (APA; Limeres-Posse et al., 2014).

La heterogeneidad de los pacientes es un sello distintivo, presentando morbilidad psiquiátrica y médica frecuentemente reportada. Los trastornos médicos reportados incluyen anomalías del sistema inmunológico, trastorno gastrointestinal, disfunción mitocondrial, trastornos del sueño y epilepsia (Dougall \& Fiske, 2008b). En el caso de comorbilidades psiquiátricas y cognitivas se pueden encontrar: Discapacidad intelectual (altamente frecuente en las personas con TEA) con una prevalencia estimada del $75 \%$; comunicación verbal y no verbal que tiende a ser limitada o inexistente (el $50 \%$ de los pacientes no adquieren lenguaje hablado); lenguaje repetitivo (a menudo) que no corresponde a un contexto, demostrando falta de comprensión; Ecolalia inmediata o tardía (repetición de una frase o palabra que ha escuchado recientemente) (presente en el $75 \%$ de los niños); Sus intereses y actividades tienden a ser limitados, y los comportamientos repetitivos son frecuentes, a menudo provocados por el estrés, la excitación o ciertos estímulos (como los ruidos). Tienden a adquirir estrictas rutinas a las que los cambios o modificaciones pueden dar lugar a actitudes resistentes. También pueden presentar una falta de coordinación motora y movimientos repetitivos del cuerpo. Una de las características principales de los niños con TEA es su bajo umbral de frustración, que con frecuencia conduce a un mal comportamiento. A medida que envejecen pueden aparecer la agitación y/o la agresividad y/ o autoagresión. Además, pueden sufrir trastornos mentales concurrentes tales como ansiedad, cambios de humor, hiperactividad, trastorno obsesivo-compulsivo o esquizofrenia (que es más frecuente durante la adolescencia). La percepción sensorial también puede verse afectada por la presentación frecuente de hipersensibilidad auditiva y táctil, reacciones exageradas a la luz y los olores, una respuesta inadecuada por parte del sistema vestibulo-visual con predominio de visión focal periférica y un umbral del dolor elevado (Luscre \& Center, 1996; Pilebro \& Bäckman, 2005; Limeres-Posse et al.).

TEA se presenta con mayor prevalencia en hombres que en mujeres 4:1 (SENADIS, Chile, 2015). La incidencia de los trastornos del espectro autista es probable que esté dentro del rango de $30-60$ casos por 10.000 , lo que significa un gran aumento sobre la es- timación original de 4 por 10.000 hace 40 años atrás (Rutter, 2005). Aunque datos más recientes son tan altas como 1 en 68, basado en niños de 8 años de edad en los Estados Unidos (CDC, 2014), otros autores hablan de 1/150-200 en 2002 (Geschwind, 2009). El aumento es en gran parte consecuencia de una mejor evaluación y una considerable ampliación del diagnóstico (Rutter).

La etiología del TEA se describe comúnmente como una predisposición genética combinada con un impacto ambiental (Newschaffer et al., 2007). La base de la investigación identifica supresiones genéticas y duplicaciones, heredadas y de novo, y las variantes raras y comunes en los TEA (Devlin \& Scherer, 2012). Se han detectado en algunos pacientes alteraciones estructurales y funcionales en el sistema límbico (responsables de las emociones y las relaciones sociales) mediante el uso de técnicas sofisticadas de imagenología. También se ha descrito la hipoactivación selectiva de ciertas áreas cerebrales asociadas con la integración motora. En los estudios realizados en familias afectadas se ha descrito un trastorno hereditario que afecta a 20 genes localizados en los cromosomas $2,7,15,16$ y 19 que interactúan entre sí (Shao et al., 2002).

Los pacientes con TEA plantean un reto a los profesionales en su tratamiento odontológico, fundamentalmente debido a los problemas de comunicación (Masi et al.).El dentista debe saber muy bien estudiado y prolijamente qué condiciones debe esperar en cada paciente y qué técnicas son las más apropiadas para su manejo: técnicas básicas (comunicación, distracción, imitación, desensibilización), técnicas físicas (restricción por parte del profesional / asistente / padres o utilizando dispositivos especializados) y técnicas avanzadas (óxido nitroso, sedación o incluso anestesia general). Estas técnicas deben ser individualizadas, teniendo en cuenta no sólo el trastorno del paciente, sino también su nivel, para tener una idea del grado de cooperación a obtener. Además, debemos analizar la patología oral del paciente, ya que la posibilidad de tratamientos largos o complejos nos puede ayudar a seleccionar la técnica más recomendable (Bartolomé-Villar et al., 2016).

Necesidad de tratamiento ortodóncico: Sólo dos estudios referentes al TEA han evaluado la necesidad de tratamiento ortodóncico pero ninguno encontró diferencias entre los dos grupos; al contrario de lo señalado por Fahlvik-Planefeldt \& Herrström, quienes estipulan que un porcentaje mayor de niños con autismo 
necesita tratamiento en comparación con el grupo control. Estos datos concuerdan con los de Luppanapornlarp et al., que encontraron un mayor número de trastornos de maloclusión (60 \%) en niños autistas, agenesias, diastemas, mordida invertida, mordida abierta y clase II molar (Bartolomé-Villar et al.).

A pesar del creciente número de niños con necesidades especiales, especialmente aquellos con trastornos neurosensoriales, se han realizado pocos estudios sobre el estado de su salud oral, comparando la población pediátrica con estas discapacidades a grupos de control sanos.

En la actualidad en Chile no existen registros nacionales de la atención ortodóncica en pacientes con NEAS ni menos programas o protocolos de atención en la especialidad que los incorporen, por lo cual se presenta esta propuesta de protocolo de atención clínica (Anexo), dirigida a especialistas en ortodoncia, en base a la trayectoria de 32 años de la Unidad de Malformación Craneofacial de la Universidad de Chile (ex I.R.M.A.D.E.M.A), que incluye experiencia clínica y trabajo en el área de malformaciones cráneo faciales, adaptada a la realidad chilena, donde el ortodoncista podrá recopilar los datos necesarios para llegar a un óptimo tratamiento en un paciente con TEA.

\section{PROTOCOLO}

Para realizar un completo estudio de un paciente con NEAS (TEA) es necesaria una acuciosa ficha clínica con énfasis en la historia clínica y una evaluación clínica con exámenes radiográficos, de modelos y fotografías; lo que nos va a permitir tener la mayor información sobre la condición presentada por cada paciente y la necesidad de tratamiento ortodóncico.

Se plantea un protocolo de evaluación de pacientes con NEAS (TEA) para su posterior evaluación, diagnóstico y tratamiento de ortopedia y ortodoncia dentomaxilar.

Historia Clínica. (Castellón et al., 2007; Bateman et al., 2010, Dougall \& Fiske, 2008b; AAPD; MINSAL, 2012).

El conocimiento de la historia clínica del paciente es esencial para evitar y disminuir el riesgo de agravar una condición médica mientras se realiza la atención odontológica. Es necesaria una historia médica precisa, completa y al día para un diagnóstico y plan de tratamiento. Se debe obtener información relativa al motivo de consulta, condiciones médicas y/o enfermedades, historia de la enfermedad actual, nombre del médico tratante, medicamentos actuales, historia social, revisión de los sistemas, alergias/sensibilidades, hospitalizaciones/cirugías, experiencias de anestesia y una completa historia odontológica si la tiene.

Si el paciente es incapaz de proporcionar información necesaria, se debe consultar con el cuidador, padres y/o con el médico tratante.

Interconsulta médica. En caso de ser necesario el odontólogo debe coordinar la atención a través de interconsultas con otros profesionales a cargo del cuidado del paciente, incluyendo médicos, enfermeras, trabajadores sociales, terapeutas. En su caso, el médico debe ser consultado con respecto a los medicamentos, sedación, anestesia general, y de restricciones especiales que puedan ser necesarias para garantizar una segura atención.

Consentimiento informado / Asentimiento. Se requiere el consentimiento informado de todos los pacientes o, en el caso de los niños, de los padres y/o cuidadores. Por lo tanto, el paciente o padre/cuidador, debe entender los riesgos y beneficios potenciales del tratamiento y legalmente aceptar estos riesgos por escrito. En el caso de menores de edad se realiza en conjunto con el consentimiento informado el asentimiento, en donde el menor expresa voluntariedad en las decisiones tomadas en dicho consentimiento, debe quedar bien documentado en la ficha clínica.

Evaluación de la complejidad del paciente. Basado en el Modelo de mezcla de casos de la Asociación Dental Británica (Bateman et al.), se utilizan ciertos criterios para determinar la complejidad de cada paciente y su posible atención, en este caso para el tratamiento ortodóncico (Tabla I).

Examen clínico (Rutter). El examen clínico debe incluir una caracterización del paciente que incluye examen extraoral e intraoral.

En cuanto a la toma de modelos, puede que por las condiciones del paciente no se pueda realizar una técnica clásica de toma de impresión, por lo que es necesario realizar modificaciones a la técnica convencional como toma de impresión con cubetas de acrílico adaptadas, uso de silicona pesada y liviana sin cubeta. 
LEIVA,V. L. \& VERGARA, S. D. Protocolo de atención ortodóncica en pacientes con trastorno del espectro autista (TEA). Int. J. Odontostomat., 11(4):399-404, 2017.

Tabla I. Evaluación de la complejidad del paciente.

PROTOCOLO DE ATENCIÓN CLINICA EN ORTODONCIA PARA PACIENTES TEA UNIDAD DE MALFORMACIONES CRANEOFACIALES UNIVERSIDAD DE CHILE.

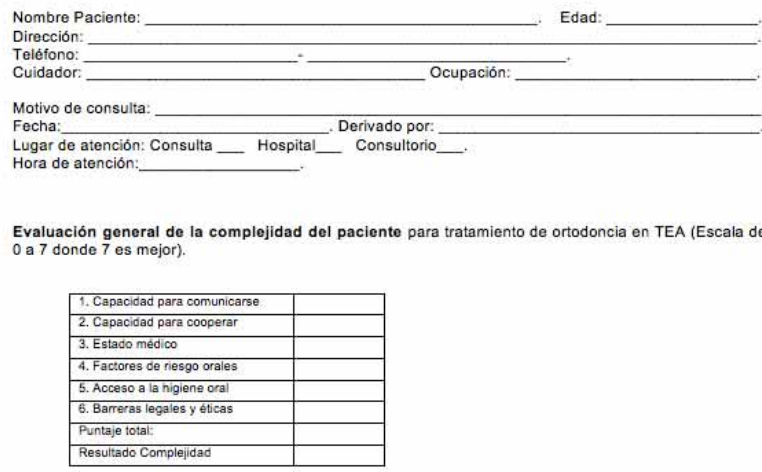

Antecedentes Médicos (Marcar con una $\mathrm{x}$ )

1. Antecedentes Familiares.

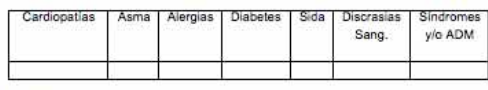

Obseraciones:

2. Antecedentes materno-infantiles.

\begin{tabular}{|l|l|l|l|l|}
\hline Embarazo & Normal & Patologia & \\
\hline Parto & De término & Premaluto & \\
\hline Nacimiento & Sano & & Paltologia & \\
\hline Lactancia / Almentación Mixta & \multicolumn{3}{|l|}{} \\
\hline
\end{tabular}

3. Enfermedades o alteraciones sistémicas.

\begin{tabular}{|c|c|}
\hline 1.Sistema Cardiovascular & 6. Enfermedades Hepáticas \\
\hline Angina & Ictericia \\
\hline Hipertensión & Hepatitis \\
\hline Antecedentes de IAM & Cirrosis \\
\hline Cingía Cardiaca & Otros: \\
\hline Cingia Valvular & 7. SNC \\
\hline Endocarditis infecciosa & Epilepsia \\
\hline Fiebre Reumática & Infartos cerebrales \\
\hline Otros: & Esclerosis mútiple \\
\hline 2. Sistema Respiratorio & Parkinson \\
\hline Asma & Parálisis cerebral \\
\hline Enfisema & Discapacidad intelectualfísica \\
\hline Tuberculosis & Otros: \\
\hline Otros: & 8. Músculo Esqueletal \\
\hline 3. Sistema Endocrino & Patologla osea \\
\hline Diabetes & Patologia muscular \\
\hline Alteracion tiroidea & Otros: \\
\hline $\begin{array}{l}\text { Alteraciones nutricionales } \\
\text { (obesidad/desnutrición) }\end{array}$ & 9. Gastrointestinal \\
\hline Otros: & Hernia hiatal \\
\hline 4. Hematología & Úlcera péptica \\
\hline Anemia & Colecistis \\
\hline Trastornos de cosgulación & Otros: \\
\hline Otros: & 10. Tejido conectivo / Dermatológico \\
\hline 5. Sistema Genito/Urinario & Lupus eritematoso sistémico \\
\hline Problemas urinarios & Esclerosis sistémica \\
\hline Enfermedad renal & Eczema \\
\hline Enfermedad de transmisión sexual & Otros: \\
\hline Otras: & $\begin{array}{l}\text { 11. Alteraciones } \quad \text { Genéticas/ } \\
\text { Sindromes } \\
\text { ¿Cuál? }\end{array}$ \\
\hline
\end{tabular}

Observaciones:

-

4. Antecedentes quirürgicos

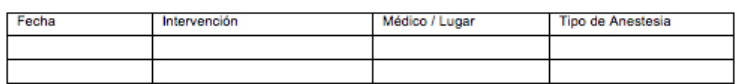

5. Medicamentos actuales

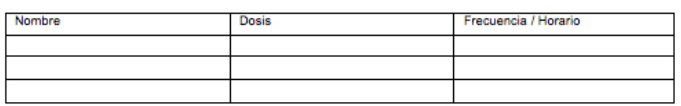

6. Antecedentes de hipersensibilidad/ alergias:

7. Grupo Sanguineo:

8. Hábitos Funcionales

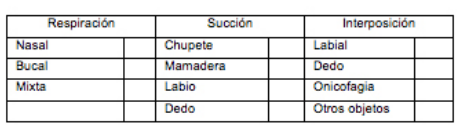

Historia social (Cuidadores / residencia / transporte / estado civil / familiares

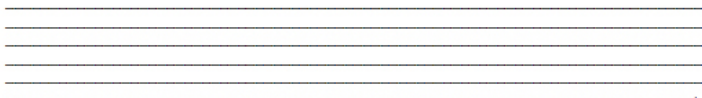

Evaluación complejidad del paciente

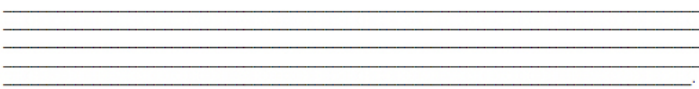

Examen clinico

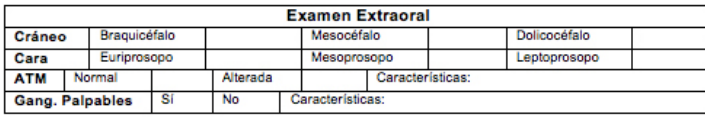

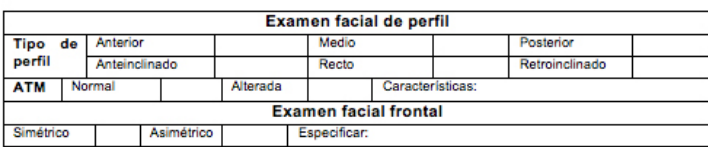

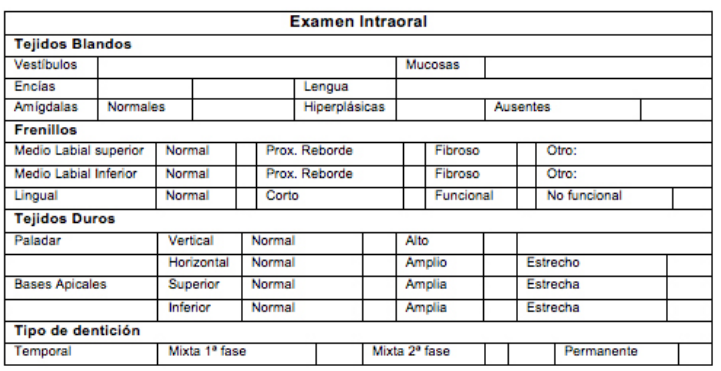

Examen Cefalométrico

\begin{tabular}{|c|c|c|c|}
\hline & \multicolumn{3}{|c|}{ Examen Cefalométrico } \\
\hline \multirow{2}{*}{$\begin{array}{ll}\begin{array}{l}\text { Tipo } \\
\text { perfil }\end{array} & \text { de }\end{array}$} & Anterior & Medio & Posterior \\
\hline & Anteinclinado & Recto & Retroinclinado \\
\hline
\end{tabular}



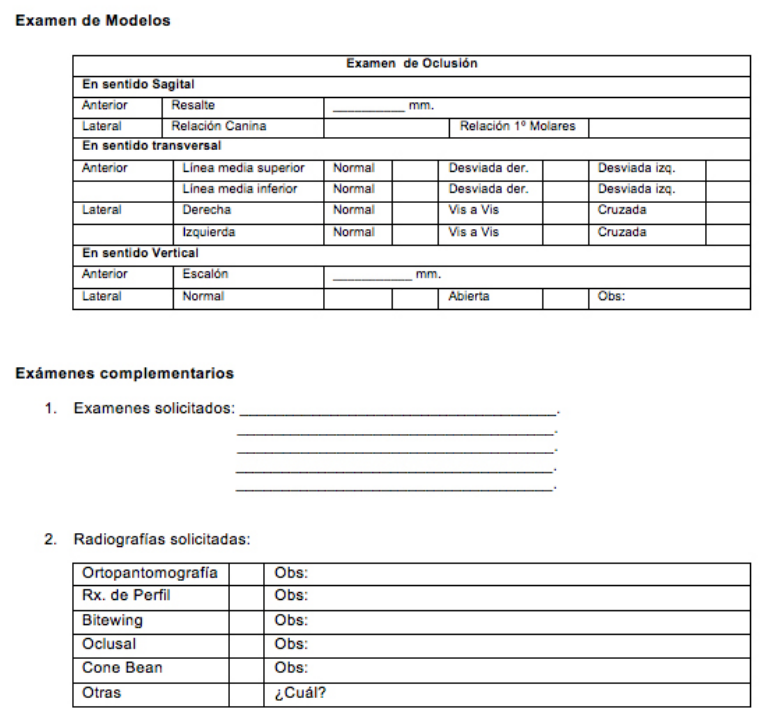

Interconsulta médica
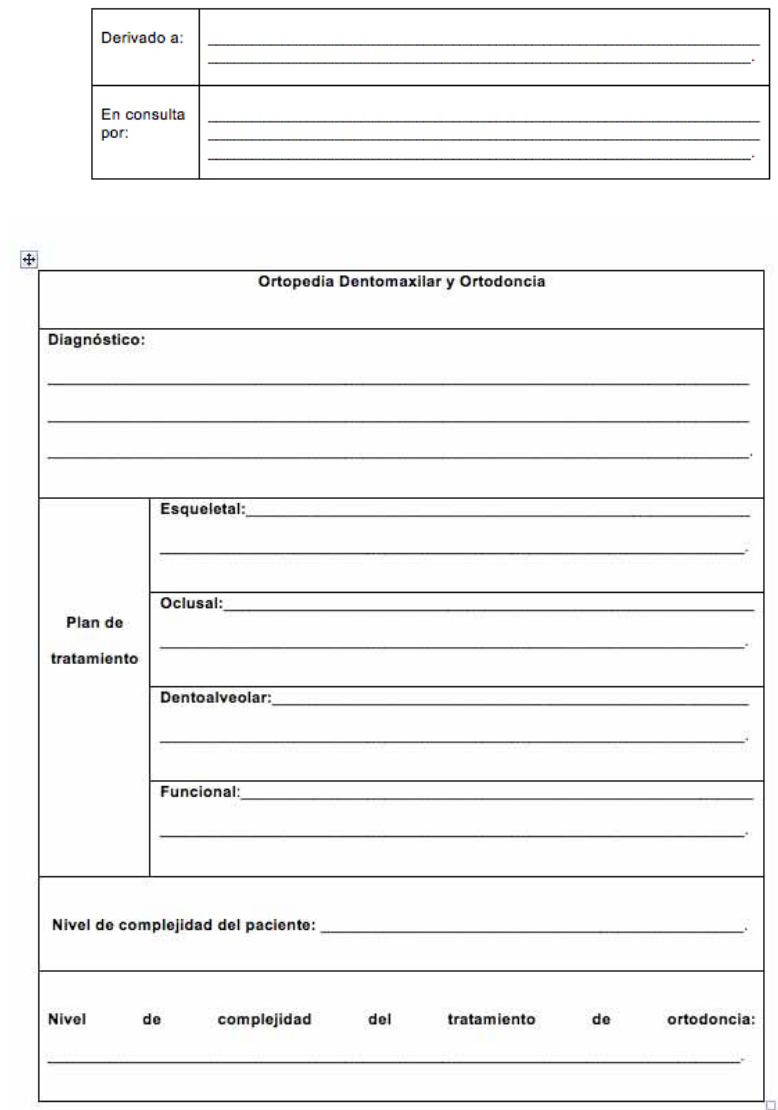

Exámenes complementarios. Los más utilizados para llegar a un buen diagnóstico ortodóncico son análisis de modelos, de fotografías, y cefalométrico, teleradiografía y radiografía panorámica y de perfil. Dependiendo del tipo de paciente con TEA, ya sea por alteraciones sensoriales, conductuales o cognitivas, en algunos pacientes no podrá ser realizada la toma de la radiografía solicitada, por lo cual es necesario adecuarse al paciente y ver la mejor opción dentro de otros tipos de radiografías ya sea periapical, periapical con deslizamiento, oclusal.

\section{CONCLUSIONES}

La historia clínica en pacientes con TEA es de gran importancia por lo que es neceario una ficha clínica completa, teniendo en cuenta también el grado de cooperación de cada paciente para obtener un buen diagnóstico y pronóstico en cuanto al tratamiento ortodóncico necesario.

El tratamiento de un paciente con TEA debe tener un enfoque multidisciplinario, ya que existirán variados profesionales de la salud trabajando en conjunto y con ello es importante mantener una fluida comunicación con el paciente y con padres/cuidadores, en el caso de no lograr una comunicación fluida con el paciente por las limitaciones comunicacionales asociadas a su trastorno.

El paciente, padres/cuidadores y el equipo odontológico deben generar una alianza tal que permita una óptima comunicación y cuidado para un mejor resultado en cada atención y en el tratamiento. El tratamiento de ortodoncia ha adquirido relevancia basado en la estética y estabilidad oclusal.

LEIVA,V. L. \& VERGARA, S. D. Orthodontic care protocol for patients with autistic spectrum disorder (ASD). Int. J. Odontostomat., 11(4):399-404, 2017.

ABSTRACT: There is currently an increase in the number of people with special oral health care needs, including those patients diagnosed with autism spectrum disorder who often require orthodontic treatment due to malocclusions. Although parents and / or caregivers are motivated to improve their quality of life by improving dento-facial aesthetics and oral function, these are also patients who do not receive treatment in conventional clinics due to behavioral problems they may present during dental care. In light of the difficulties that can be encountered during clinical dental care, the present study presents a protocol of orthodontic care in patients with autism spectrum disorder. The protocol is useful in that it allows an adequate diagnosis, treatment plan and prognosis in the orthodontic specialty required by each patient.

KEY WORDS: special needs, autism spectrum disorder, protocol, orthodontics. 


\section{REFERENCIAS BIBLIOGRÁFICAS}

Abrahams, B. S. \& Geschwind, D. H. Advances in autism genetics: on the threshold of a new neurobiology. Nat. Rev. Genet., 9(5):341-55, 2008.

American Academy of Pediatric Dentistry (AAPD). Council on Clinical Affairs. Guideline on management of dental patients with special health care needs. Pediatr. Dent., 34(5):160-5, 2012.

American Psychiatric Association (APA). Diagnostic and Statistical Manual of Mental Disorders. $5^{\text {th }}$ ed. Washington D. C., American Psychiatric Association, 2013.

Bartolomé-Villar, B.; Mourelle-Martínez, M. R.; DiéguezPérez, M. \& de Nova-García, M. J. Incidence of oral health in paediatric patients with disabilities: Sensory disorders and autism spectrum disorder. Systematic review II. J. Clin. Exp. Dent., 8(3):e344-51, 2016.

Bateman, P.; Arnold, C.; Brown, R.; Foster, L. V.; Greening, S.; Monaghan, N. \& Zoitopoulos, L. BDA special care case mix model. Br. Dent. J., 208(7):291-6, 2010.

Castellón, L.; Uribe, F.; Leiva, N.; Agurto, P. \& Fariña, R. Protocolo ortodóncico-quirúrgico para injertos alveolares secundarios en pacientes portadores de fisura labioalveolopalatina. Rev. Chil. Ortod., 24(2):92-102, 2007.

Center for Disease Control and Prevention (CDC). Prevalence of autism spectrum disorder among children aged 8 years - Autism and developmental disabilities monitoring network, 11 sites, United States, 2010. Morb. Mortal. Wkly. Rep. Surveill. Summ., 63(2):1-14, 2014.

Devlin, B. \& Scherer, S. W. Genetic architecture in autism spectrum disorder. Curr. Opin. Genet. Dev., 22(3):22937, 2012.

Dougall, A. \& Fiske, J. Access to special care dentistry, part 1. Access. Br. Dent. J., 204(11):605-16, 2008 a.

Dougall, A. \& Fiske, J. Access to special care dentistry, part 3. Consent and capacity. Br. Dent. J., 205(2):71-81, $2008 \mathrm{~b}$.

Geschwind, D. H. Advances in autism. Annu. Rev. Med., 60:367-80, 2009.

Limeres-Posse, J.; Castaño-Novoa, P.; Abeleira-Pazos, M. \& Ramos-Barbosa, I. Behavioural aspects of patients with Autism Spectrum Disorders (ASD) that affect their dental management. Med. Oral Patol. Oral Cir. Bucal,19(5):e46772, 2014.

Luscre, D. M. \& Center, D. B. Procedures for reducing dental fear in children with autism. J. Autism Dev. Disord., 26(5):547-56, 1996.

Masi, A.; Demayo, M. M.; Glozier, N. \& Guastella, A. J. An overview of autism spectrum disorder, heterogeneity and treatment options. Neurosci. Bull., 33(2):183-93, 2017.

Ministerio de Salud (MINSAL). Salud Oral Integral para Menores de 20 Años en Situación de Discapacidad que Requieren Cuidados Especiales en Odontología. Santiago de Chile, Ministerio de Salud, Gobierno de Chile, 2012.

Newschaffer, C. J.; Croen, L. A.; Daniels, J.; Giarelli, E.; Grether, J. K.; Levy, S. E.; Mandell, D. S.; Miller, L. A.; Pinto-Martin, J.; Reaven, J.; Reynolds, A. M.; Rice, C. E.;
Schendel, D. \& Windham, G. C. The epidemiology of autism spectrum disorders. Annu. Rev. Public Health, 28:235-58, 2007.

Pilebro, C. \& Bäckman, B. Teaching oral hygiene to children with autism. Int. J. Paediatr. Dent., 15(1):1-9, 2005.

Rutter, M. Incidence of autism spectrum disorders: changes over time and their meaning. Acta Paediatr., 94(1):2-15, 2005.

Servicio Nacional de la Discapacidad (SENADIS), Instituto Nacional de Estadísticas. Segundo Estudio Nacional de la Discapacidad en Chile. Santiago de Chile, Ministerio de Salud, Gobierno de Chile, 2015.

Shao, Y.; Wolpert, C. M.; Raiford, K. L.; Menold, M. M.; Donnelly, S. L.; Ravan, S. A.; Bass, M. P.; McClain, C.; von Wendt, L.; Vance, J. M.; Abramson, R. H.; Wright, H. H.; Ashley-Koch, A.; Gilbert, J. R.; DeLong, R. G.; Cuccaro, M. L. \& Pericak-Vance, M. A. Genomic screen and follow-up analysis for autistic disorder. Am. J. Med. Genet., 114(1):99-105, 2002.

Dirección para correspondencia

Noemí LeivaVillagra

General Holley \#2381 of. 606 B

Providencia

Santiago

CHILE

Email: leivanoemi@yahoo.com

Recibido : 29-05-2017

Aceptado: 05-09-2017 